Rational values of Weierstrass zeta functions

Jones, Gareth O. and Thomas, Margaret E.M. 2014

MIMS EPrint: 2015.33

Manchester Institute for Mathematical Sciences

School of Mathematics

The University of Manchester

\footnotetext{
Reports available from: http://eprints.maths.manchester.ac.uk/

And by contacting: The MIMS Secretary

School of Mathematics

The University of Manchester

Manchester, M13 9PL, UK
} 


\title{
Rational values of Weierstrass zeta functions
}

\author{
Gareth O. Jones* and Margaret E. M. Thomas ${ }^{\dagger}$
}

May 24, 2015

\begin{abstract}
We answer a question of Masser by showing that for the Weierstrass zeta function $\zeta$ corresponding to a given lattice $\Lambda$, the density of algebraic points of absolute multiplicative height bounded by $T$ and degree bounded by $k$ lying on the graph of $\zeta$, restricted to an appropriate domain, does not exceed $c(\log T)^{15}$, for an effective constant $c>0$ depending on $k$ and on $\Lambda$. Using different methods, we also give two bounds of the same form for the density of algebraic points of bounded height in a fixed number field lying on the graph of $\zeta$ restricted to an appropriate subset of $(0,1)$. In one case the constant $c$ can be shown not to depend on the choice of lattice; in the other, the exponent can be improved to 12 .
\end{abstract}

Keywords: Weierstrass zeta functions, counting, irrationality

2010 Mathematics Subject Classification:

Primary: 11J72. Secondary: 03C64, 33E05.

\section{Introduction}

In [Mas11], Masser proves the following bound on the density of rational points on the graph of the Riemann zeta function.

Fact 1.1 ([Mas11, Theorem, p. 2038]). There is a positive effective absolute constant $C$ such that, for any integer $D \geq 3$, the number of rational $z$ with $2<$ $z<3$ of denominator at most $D$ such that $\zeta(z)$ is rational also of denominator at most $D$ is at most $C\left(\frac{\log D}{\log \log D}\right)^{2}$.

In order to arrive at this statement, he in fact proves the following more general result concerning the density of algebraic points of bounded degree.

Fact 1.2 ([Mas11, p. 2045]). There is an absolute constant $c>0$ such that, for any integers $k, T \geq 1$, there are at most

$$
c\left(\frac{k^{2} \log 4 T}{\log (k \log 4 T)}\right)^{2}
$$

different complex numbers $z$ with $\left|z-\frac{5}{2}\right| \leq \frac{1}{2}$ such that $[\mathbb{Q}(z, \zeta(z)): \mathbb{Q}] \leq k$, $H(z) \leq T$ and $H(\zeta(z)) \leq T$, where $H$ is the absolute non-logarithmic height. UK

*School of Mathematics, University of Manchester, Oxford Road, Manchester M13 9PL,

${ }^{\dagger}$ Zukunftskolleg, Fachbereich Mathematik und Statistik, Fach 216, Universitätsstraße 10, Universität Konstanz, D-78457 Konstanz, Germany 
In the context of outlining these results, Masser states that "It may be an interesting problem to prove analogues of our theorem for other natural functions. For example the Euler gamma function $\Gamma(z)$, about which we know even fewer irrationality properties. Or the Weierstrass zeta function $\zeta(z)$ seems promising, say with rational invariants; in spite of its differential equation we do not know a single rational $z$ with $\zeta(z)$ irrational. Jonathan Pila has also suggested $\frac{\zeta(z)}{\pi^{z}}$ [for $\zeta$ the Riemann zeta function]." The functions $\Gamma(z)$ and $\frac{\zeta(z)}{\pi^{z}}$ (for $\zeta$ the Riemann zeta function) were analysed by the first author and Boxall in [BJ15], where results analogous to Fact 1.2 were proved for which the exponent is $3+\epsilon$ in place of 2 , although they hold for the restriction to $(2, \infty)$. Independently, Besson adapted Masser's methods and proved that a bound $C(n) \frac{\left(k^{2} \log T\right)^{2}}{\log (k \log T)}$ holds in the case of $\Gamma(z)$ restricted to any interval $[n-1, n]$ (see [Bes14]).

It is the aim of this note to address the question of the Weierstrass zeta function, to which the methods of [BJ15] and [Bes14] do not apply. We will provide three bounds of a similar nature using different methods, all of which apply to Weierstrass zeta functions in general (and do not require that they have rational invariants). Our main result (in Section 4) corresponds to Fact 1.2 above. We also obtain two bounds for the density of points in a fixed number field on the graph of the form $\left.\zeta\right|_{I}$, for an interval $I \subseteq \mathbb{R}$. In all cases the bound has the form $c(\log T)^{\gamma}$, but the value of $\gamma$ and the uniformity of the constant $c$ differ in each case. (In all cases an effective constant $c$ can be found.) In our main result and our first restricted case, the constant $c$ depends both on the lattice and on $k$, where $k$ is, respectively, the maximum degree of the points being counted or the degree of the number field. However, in our second restricted case, a constant $c$ can be found which depends only on $k$, at the cost of a much larger exponent $\gamma$ than is obtained in the other cases.

In order to state these results, we must first fix the following notation. Let $H$ be the absolute multiplicative height. For rational numbers, this is given by $H\left(\frac{a}{b}\right)=\max \{|a|, b\}$, where $a, b \in \mathbb{Z}, \operatorname{gcd}(a, b)=1$ and $b>0$. For algebraic numbers $\beta$, it is given by

$$
H(\beta)=\left(\left|a_{0}\right| \prod_{i=1}^{d} \max \left\{\left|\beta_{i}\right|, 1\right\}\right)^{\frac{1}{d}}
$$

where $m_{\beta}(Z)=a_{0}+a_{1} Z+\ldots+a_{d} Z^{d}=a_{0}\left(Z-\beta_{1}\right) \cdots\left(Z-\beta_{d}\right) \in \mathbb{Z}[Z]$ is a minimum polynomial of $\beta$ with $\operatorname{gcd}\left(a_{0}, \ldots, a_{d}\right)=1$. This agrees with the previous definition of $H(x)$ for $x$ rational. (For more details see [BG06, p. 16].) We extend $H$ to tuples of real numbers $\left(\alpha_{1}, \ldots, \alpha_{n}\right)$ by setting $H\left(\left(\alpha_{1}, \ldots, \alpha_{n}\right)\right):=$ $\max _{1 \leq i \leq n}\left\{H\left(\alpha_{i}\right)\right\}$. Then, for $\mathcal{W} \subseteq \mathbb{C}^{n}, F$ a fixed number field of degree $k$ and $T$ a positive real number, we set

$$
\begin{aligned}
\mathcal{W}(F, T) & =\left\{\bar{z} \in \mathcal{W} \cap F^{n} \mid H(\bar{z}) \leq T\right\} \\
N_{F}(\mathcal{W}, T) & =\# \mathcal{W}(F, T) .
\end{aligned}
$$

For an arbitrary degree $k \in \mathbb{N}$, we set

$$
\begin{aligned}
\mathcal{W}(k, T) & =\{\bar{z} \in \mathcal{W} \mid[\mathbb{Q}(\bar{z}): \mathbb{Q}] \leq k, H(\bar{z}) \leq T\} \\
N_{k}(\mathcal{W}, T) & =\# \mathcal{W}(k, T) .
\end{aligned}
$$


Our three results may be stated collectively as follows. We give the definition of a Weierstrass zeta function and all the related terminology required in Section 3 .

Theorem 1.3. Let $\zeta_{\Lambda}: \mathbb{C} \backslash \Lambda \rightarrow \mathbb{C}$ be the Weierstrass zeta function corresponding to a fixed lattice $\Lambda \subseteq \mathbb{C}$ with generators $\omega_{1}, \omega_{2}$ (chosen so that $\left|\omega_{1}\right|=$ $\min _{\omega \in \Lambda}\{|\omega|\}$, and $\left.\left|\omega_{2}\right|=\min _{\omega \in \Lambda \backslash \mathbb{Z} \omega_{1}}\{|\omega|\}\right)$. Set $\mathcal{F}$ to be the fundamental domain with corners $0, \omega_{1}, \omega_{2}$ and $\omega_{1}+\omega_{2}$.

(i) Fix $k \in \mathbb{N}$. There exist effectively computable numbers $R\left(\omega_{1}, \omega_{2}\right)>0$ and $c\left(k, \omega_{1}, \omega_{2}\right)>0$ such that

$$
N_{k}\left(\mathcal{W}_{1}, T\right) \leq c \cdot(\log T)^{15}
$$

where $\mathcal{W}_{1}$ is the graph of $\zeta_{\Lambda} \uparrow_{\Delta_{R}}$, and $\Delta_{R}:=\left\{z \in \mathbb{C}|| z-\frac{\omega_{1}+\omega_{2}}{4} \mid \leq R\right\} \subseteq$ $\mathcal{F}$.

Now suppose that $F \subseteq \mathbb{C}$ is a number field of degree $k \in \mathbb{N}$.

(ii) Let $B$ be a compact subset of $(0,1) \cap \mathcal{F} \backslash(\Lambda / 2)$. There exists an effective constant $c\left(k, \omega_{1}, \omega_{2}, B\right)>0$ such that

$$
N_{F}\left(\mathcal{W}_{2}, T\right) \leq c \cdot(\log T)^{12}
$$

where $\mathcal{W}_{2}$ is the graph of $\left.\zeta_{\Lambda}\right\rceil_{B}$.

(iii) Set $I:=\mathcal{F} \cap \mathbb{R}$. There exists an effective constant $c(k)>0$ such that

$$
N_{F}\left(\mathcal{W}_{3}, T\right) \leq c \cdot(\log T)^{41},
$$

where $\mathcal{W}_{3}$ is the graph of $\left.\zeta_{\Lambda}\right\rceil_{I}$.

Remark. Cases (ii) and (iii) only give a non-trivial statement in the event that $\mathcal{F} \cap(0,1)$, respectively $\mathcal{F} \cap \mathbb{R}$, is non-empty.

In proving all three cases we shall use the fact that Weierstrass zeta functions may be defined implicitly from certain Pfaffian functions, a property proved by Macintyre [Mac08]. Pfaffian functions are derived from solutions to particular triangular systems of polynomial differential equations; we shall outline the required theory of these functions in Section 2. Then, in Section 3, we shall show how any Weierstrass zeta function may be represented using them. The bounds will follow in the remaining sections; we first prove the general statement (i) in Section 4, followed by proofs of statements (ii) and (iii) of the special setting in Section 5. Although all three results follow rather straightforwardly from the literature, this concrete setting allows us the opportunity to compare the different methods in each case.

\section{Acknowledgements}

First author supported in part by UK Engineering and Physical Sciences Research Council grants EP/J01933X/1 ("O-minimality and diophantine geometry"), EP/E050441/1 ("The Manchester Centre for Interdisciplinary Computational and Dynamical Analysis" (CICADA)), and EP/F043236/1 (EPSRC Postdoctoral Fellowship). Second author supported in part by the USA National Science Foundation under Grant No. 0932078000 while in residence at the Mathematical Sciences Research Institute in Berkeley, California, USA, during the Spring 2014 semester ("Model Theory, Arithmetic Geometry and Number Theory"), and by the Zukunftskolleg, Universität Konstanz, Germany. 


\section{Pfaffian functions}

Here we outline the necessary aspects of the general theory of Pfaffian functions that we shall use in the proofs in the later sections. We follow the presentation of [GV04].

Definition 2.1. A sequence $f_{1}, \ldots, f_{r}: U \rightarrow \mathbb{R}$ of analytic functions on an open set $U \subseteq \mathbb{R}^{n}$ is said to be a Pfaffian chain of order $r$ and degree $\alpha$ if there are polynomials $P_{i, j} \in \mathbb{R}\left[X_{1}, \ldots, X_{n+j}\right]$ of degree at most $\alpha$ such that

$$
\frac{\partial f_{j}}{\partial x_{i}}=P_{i, j}\left(\bar{x}, f_{1}(\bar{x}), \ldots, f_{j}(\bar{x})\right) \quad \text { for all } i=1, \ldots, r \text { and } j=1, \ldots, n \text {. }
$$

Given such a chain, we say that a function $f: U \rightarrow \mathbb{R}$ is Pfaffian of order $r$ and degree $(\alpha, \beta)$, with chain $f_{1}, \ldots, f_{r}$, if there is a polynomial $P \in$ $\mathbb{R}\left[X_{1}, \ldots, X_{n}, Y_{1}, \ldots, Y_{r}\right]$ of degree at most $\beta$ such that $f(\bar{x})=P\left(\bar{x}, f_{1}(\bar{x}), \ldots, f_{r}(\bar{x})\right)$, for all $\bar{x} \in U$.

We denote by $\mathbb{R}_{\text {Pfaff }}$ the expansion of the real ordered field by all Pfaffian functions $f: \mathbb{R}^{n} \rightarrow \mathbb{R}$, for $n \geq 1$.

Definition 2.2. Let $g: U \rightarrow \mathbb{R}$, with $U \subseteq \mathbb{R}^{m}$, be definable in this structure. Following [JW08], we say that $g$ is implicitly defined by Pfaffian functions if there exist $n \geq 1$, Pfaffian functions $p_{1}, \ldots, p_{n}: \mathbb{R}^{m+n} \rightarrow \mathbb{R}$ and definable smooth functions $g_{1}, \ldots, g_{n}: U \rightarrow \mathbb{R}$ such that $g_{1}=g$ and

$$
\begin{array}{r}
p_{1}\left(\bar{x}, g_{1}(\bar{x}), \ldots, g_{n}(\bar{x})\right)=\cdots=p_{n}\left(\bar{x}, g_{1}(\bar{x}), \ldots, g_{n}(\bar{x})\right)=0, \\
\operatorname{det}\left(\frac{\partial\left(p_{1}, \ldots, p_{n}\right)}{\partial\left(x_{n+1}, \ldots, x_{n+m}\right)}\right)\left(\bar{x}, g_{1}(\bar{x}), \ldots, g_{n}(\bar{x})\right) \neq 0,
\end{array}
$$

for all $\bar{x} \in U$. Moreover, we say that $g$ has an implicit definition of complexity $(n, r, \alpha, \beta)$ if the functions $p_{1}, \ldots, p_{n}$ have a common chain of order $r$, and degree at most $(\alpha, \beta)$.

We conclude this short section with the following theorem, which is central to the theory of Pfaffian functions and to our analysis in the subsequent sections. It is Khovanskii's theorem bounding the number of connected components of a Pfaffian variety ([Kho80],[Kho91]).

Fact 2.3 (Khovanskii, see $\left[\right.$ GV04, 3.3]). Suppose that $p_{1}, \ldots, p_{k}: \mathbb{R}^{n} \rightarrow \mathbb{R}$ are Pfaffian functions, with a common chain of order $r$, and degree at most $(\alpha, \beta)$. Then the variety $V\left(p_{1}, \ldots, p_{k}\right)=\left\{\bar{x} \in \mathbb{R}^{n} \mid p_{1}(\bar{x})=\cdots=p_{k}(\bar{x})=0\right\}$ has at most

$$
2^{\frac{r(r-1)}{2}+1} \beta(\alpha+2 \beta-1)^{n-1}((2 n-1)(\alpha+\beta)-2 n+2)^{r}
$$

connected components.

\section{Weierstrass functions}

In this section we will gather together some general theory of Weierstrass functions and apply it in identifying the Pfaffian complexity of the real and imaginary parts of Weierstrass zeta functions. 


\subsection{General theory of Weierstrass functions}

Let $\Lambda$ be a lattice in the complex plane $\mathbb{C}$. We consider it as being generated by periods $\omega_{1}$ and $\omega_{2}$, where $\omega_{1}$ is an element of $\Lambda \backslash\{0\}$ with smallest $\left|\omega_{1}\right|$, and $\omega_{2}$ is an element of $\Lambda \backslash \mathbb{Z} \omega_{1}$ with smallest $\left|\omega_{2}\right|$, so $\left|\omega_{1}\right| \leq\left|\omega_{2}\right|$ and $\Lambda:=$ $\left\{m \omega_{1}+n \omega_{2} \mid m, n \in \mathbb{Z}\right\}$. The Weierstrass elliptic function $\wp_{\Lambda}: \mathbb{C} \backslash \Lambda \rightarrow \mathbb{C}$ corresponding to $\Lambda$ is defined as follows:

$$
\wp_{\Lambda}(z):=\frac{1}{z^{2}}+\sum_{0 \neq \omega \in \Lambda}\left(\frac{1}{(z-\omega)^{2}}-\frac{1}{\omega^{2}}\right) .
$$

It has poles at the points of $\Lambda$ and its Laurent series expansion is given by

$$
\wp_{\Lambda}(z)=\frac{1}{z^{2}}+\frac{g_{2}}{20} z^{2}+\frac{g_{3}}{28} z^{4}+O\left(z^{6}\right)
$$

for invariants $g_{2}, g_{3}$. Moreover, it satisfies the differential equation

$$
\left(\wp_{\Lambda}^{\prime}(z)\right)^{2}=g_{\Lambda}\left(\wp_{\Lambda}(z)\right)
$$

where $g_{\Lambda}(z) \in \mathbb{C}[Z]$ is the polynomial given by $4 z^{3}-g_{2} z-g_{3}$. On any fundamental domain $\mathcal{F}$ (for example, we will fix $\mathcal{F}$ to be the parallelogram with corners $0, \omega_{1}, \omega_{2}$ and $\omega_{1}+\omega_{2}$, including the lines $\left[0, \omega_{1}\right]$ and $\left.\left[0, \omega_{2}\right]\right)$, the function $\wp_{\Lambda}\left\lceil\mathcal{F}: \mathcal{F} \rightarrow \mathbb{C}\right.$ has a well-defined inverse function $\wp_{\Lambda}^{-1}$, which has two branches at any point $w$ for which $g_{\Lambda}(w) \neq 0$. This inverse function satisfies the Weierstrass integral of the first kind

$$
\left(\wp_{\Lambda}^{-1}\right)(z)=\int^{z} \frac{d w}{\sqrt{g_{\Lambda}(w)}} .
$$

The Weierstrass zeta function $\zeta_{\Lambda}: \mathbb{C} \backslash \Lambda \rightarrow \mathbb{C}$ corresponding to $\Lambda$ may then be defined by the following:

$$
\zeta_{\Lambda}(z):=\frac{1}{z}+\sum_{0 \neq \omega \in \Lambda}\left(\frac{1}{z-\omega}+\frac{1}{\omega}+\frac{z}{\omega^{2}}\right) .
$$

Its derivative is the negation of $\wp_{\Lambda}$, i.e. for all $z \in \mathcal{F}$,

$$
\zeta_{\Lambda}^{\prime}(z)=-\wp_{\Lambda}(z)
$$

We may also express the relationship between $\wp_{\Lambda}$ and $\zeta_{\Lambda}$ in the following way:

$$
\zeta_{\Lambda}(z)=-G_{\Lambda}\left(\wp_{\Lambda}(z)\right)
$$

where $G_{\Lambda}$ is given by the Weierstrass integral of the second kind

$$
G_{\Lambda}(z)=\int^{z} \frac{w d w}{\sqrt{g_{\Lambda}(w)}} .
$$

For more details, see [DV73, $§ 443,50]$. 


\subsection{Implicitly defining $\zeta_{\Lambda}$ from Pfaffian functions}

Our aim here is to provide the details of the way in which $\zeta_{\Lambda}$ may be implicitly defined from Pfaffian functions. We begin by identifying $\mathbb{C}$ with $\mathbb{R}^{2}$ in the usual way, so $z=x+i y$. For this subsection, we let the notation $\bar{z}$ denote the complex conjugate of $z$, for any $z \in \mathbb{C}$. We will provide the proof of the following lemma, which is a version of Theorems 2.4 and 3.1 of [Mac08], in which we include all of the details required in the later sections.

Lemma 3.1. For each $w \in \mathbb{C}$ with $g_{\Lambda}(w) \neq 0$ and each analytic branch of $\sqrt{g_{\Lambda}(z)}$ on an open, simply connected neighbourhood $U \subseteq \mathbb{C}$ of $w$ on which $g_{\Lambda}$ does not vanish, the real and imaginary parts of the corresponding branches of $\wp_{\Lambda}^{-1}: U \rightarrow \mathbb{C}$ and $G_{\Lambda}: U \rightarrow \mathbb{C}$ are real Pfaffian functions of order 6 and degree $(9,1)$. Collectively, they are real Pfaffian with order 9 and degree $(9,1)$.

Proof. Let us fix both $w \in \mathbb{C}$ and an analytic branch of $\sqrt{g_{\Lambda}(z)}$ on some open, simply connected neighbourhood $U$ of $g_{\Lambda}(w)$, so that we are considering a fixed (corresponding) branch of each of $\wp_{\Lambda}^{-1}$ and $G_{\Lambda}$ on $U$. Macintyre proved the statement for $\operatorname{Re}\left(\wp_{\Lambda}^{-1}\right), \operatorname{Im}\left(\wp_{\Lambda}^{-1}\right): U \rightarrow \mathbb{R}$ in $[\mathrm{Mac} 08,2.2,2.4]$. He also indicated that, in order to prove it for $\operatorname{Re}\left(G_{\Lambda}\right)$ and $\operatorname{Im}\left(G_{\Lambda}\right)$, one follows exactly the same argument [Mac08, 3.1]. However, we shall include the details here in order to identify the Pfaffian chain in each case, and hence demonstrate the order and degree, which will be used in later arguments.

We begin by considering the Cauchy-Riemann equations for $\wp_{\Lambda}^{-1}$ and $G_{\Lambda}$. In order to simplify the notation we let $u:=\operatorname{Re}\left(\wp_{\Lambda}^{-1}\right), v:=\operatorname{Im}\left(\wp_{\Lambda}^{-1}\right), \tilde{u}:=\operatorname{Re}\left(G_{\Lambda}\right)$ and $\tilde{v}:=\operatorname{Im}\left(G_{\Lambda}\right)$. Then, using identities 3.0.1 and 3.0.3, we see that

$$
\begin{aligned}
& \frac{\partial u}{\partial x}=\frac{\partial v}{\partial y}=\frac{1}{2}\left(\frac{\partial}{\partial z} \wp_{\Lambda}^{-1}(z)+\overline{\frac{\partial}{\partial z} \wp_{\Lambda}^{-1}(z)}\right)=\frac{\operatorname{Re}\left(\sqrt{g_{\Lambda}(z)}\right)}{\left|g_{\Lambda}(z)\right|} \\
& \frac{\partial u}{\partial y}=-\frac{\partial v}{\partial x}=-\frac{1}{2 i}\left(\frac{\partial}{\partial z} \wp_{\Lambda}^{-1}(z)-\overline{\frac{\partial}{\partial z} \wp_{\Lambda}^{-1}(z)}\right)=\frac{\operatorname{Im}\left(\sqrt{g_{\Lambda}(z)}\right)}{\left|g_{\Lambda}(z)\right|} \\
& \frac{\partial \tilde{u}}{\partial x}=\frac{\partial \tilde{v}}{\partial y}=\frac{1}{2}\left(\frac{\partial}{\partial z} G_{\Lambda}(z)+\frac{\partial}{\partial z} G_{\Lambda}(z)\right)=\frac{\operatorname{Re}\left(\bar{z} \sqrt{g_{\Lambda}(z)}\right)}{\left|g_{\Lambda}(z)\right|} \\
& \frac{\partial \tilde{u}}{\partial y}=-\frac{\partial \tilde{v}}{\partial x}=-\frac{1}{2 i}\left(\frac{\partial}{\partial z} G_{\Lambda}(z)-\frac{\partial}{\partial z} G_{\Lambda}(z)\right)=\frac{\operatorname{Im}\left(\bar{z} \sqrt{g_{\Lambda}(z)}\right)}{\left|g_{\Lambda}(z)\right|}
\end{aligned}
$$

Now let us observe that the polynomial $g_{\Lambda}(z)$, as a function of the variables $x$ and $y$, can be written as

$$
g_{\Lambda}(z)=A_{\Lambda}(x, y)+i B_{\Lambda}(x, y)
$$

where $A_{\Lambda}$ and $B_{\Lambda}$ are polynomials of degree 3 over $\mathbb{Q}\left(\operatorname{Re}\left(g_{2}\right), \operatorname{Im}\left(g_{2}\right), \operatorname{Re}\left(g_{3}\right), \operatorname{Im}\left(g_{3}\right)\right)$. Alternatively, we may write

$$
g_{\Lambda}(z)=r e^{i \theta}
$$

from which we observe that

$$
\sqrt{g_{\Lambda}(z)}= \pm r^{\frac{1}{2}} e^{i \frac{\theta}{2}}
$$


Combining 3.1.5 and 3.1.6, and assuming that we take the positive square root of $r=\sqrt{A_{\Lambda}^{2}+B_{\Lambda}^{2}}$, we establish the following identities:

$$
\begin{aligned}
\operatorname{Re}\left(\sqrt{g_{\Lambda}(z)}\right) & =\frac{1}{\sqrt{2}} \sqrt{\sqrt{A_{\Lambda}^{2}+B_{\Lambda}^{2}}+A_{\Lambda}} \\
\operatorname{Im}\left(\sqrt{g_{\Lambda}(z)}\right) & =\frac{1}{\sqrt{2}} \sqrt{\sqrt{A_{\Lambda}^{2}+B_{\Lambda}^{2}}-A_{\Lambda}} \\
\operatorname{Re}\left(\bar{z} \sqrt{g_{\Lambda}(z)}\right) & =\frac{1}{\sqrt{2}}\left(x \sqrt{\sqrt{A_{\Lambda}^{2}+B_{\Lambda}^{2}}+A_{\Lambda}}+y \sqrt{\sqrt{A_{\Lambda}^{2}+B_{\Lambda}^{2}}-A_{\Lambda}}\right) \\
\operatorname{Im}\left(\bar{z} \sqrt{g_{\Lambda}(z)}\right) & =\frac{1}{\sqrt{2}}\left(x \sqrt{\sqrt{A_{\Lambda}^{2}+B_{\Lambda}^{2}}+A_{\Lambda}}-y \sqrt{\sqrt{A_{\Lambda}^{2}+B_{\Lambda}^{2}}-A_{\Lambda}}\right)
\end{aligned}
$$

These would be purely formal without having already made a choice of branch of $\pm \sqrt{g_{\Lambda}(z)}$, which determines the signs of $\sqrt{\sqrt{A_{\Lambda}^{2}+B_{\Lambda}^{2}}+A_{\Lambda}}$ and $\sqrt{\sqrt{A_{\Lambda}^{2}+B_{\Lambda}^{2}}-A_{\Lambda}}$.

Now let us define the following functions on $U$.

$$
\begin{aligned}
f_{1} & =\frac{1}{\sqrt{A_{\Lambda}^{2}+B_{\Lambda}^{2}}} \\
f_{2} & =\frac{1}{\sqrt{\sqrt{A_{\Lambda}^{2}+B_{\Lambda}^{2}}+A_{\Lambda}}} \\
f_{3} & =\frac{1}{\sqrt{\sqrt{A_{\Lambda}^{2}+B_{\Lambda}^{2}}-A_{\Lambda}}} \\
f_{4} & =\sqrt{\sqrt{A_{\Lambda}^{2}+B_{\Lambda}^{2}}+A_{\Lambda}} \\
f_{5} & =\sqrt{\sqrt{A_{\Lambda}^{2}+B_{\Lambda}^{2}}-A_{\Lambda}}
\end{aligned}
$$

If we also set $f_{6}=u$, then the functions $f_{1}, \ldots, f_{6}$ form a Pfaffian chain. It is easy to compute that it has degree 9. Consequently, $u$ is Pfaffian of order 6 and degree $(9,1)$. The same argument applies if we set $f_{6}$ to be any of $v, \tilde{u}$ or $\tilde{v}$. This proves the first statement. Moreover, $u, v, \tilde{u}$ and $\tilde{v}$ taken collectively are Pfaffian with common chain $f_{1}, \ldots, f_{5}, u, v, \tilde{u}, \tilde{v}$, and have therefore common order 9 and degree $(9,1)$.

Combining Lemma 3.1 with 3.0.2 we see the following.

Corollary 3.2. The functions $\operatorname{Re}\left(\zeta_{\Lambda}\right)$ and $\operatorname{Im}\left(\zeta_{\Lambda}\right)$, when restricted to a suitable set $U \subseteq \mathcal{F} \backslash(\Lambda / 2) \subseteq \mathbb{R}^{2} \backslash \Lambda$, may be implicitly defined by Pfaffian functions, with complexity of definition $(3,8,9,1)$.

Proof. For the corresponding branches of $\wp_{\Lambda}^{-1}$ and $G_{\Lambda}$, we may write

$$
\begin{aligned}
\operatorname{graph}\left(\operatorname{Re}\left(\zeta_{\Lambda}\right)\right)=\left\{(x, y, w) \in U \times \mathbb{R} \mid \exists t_{1}, t_{2}\right. & \left(x-\operatorname{Re}\left(\wp_{\Lambda}^{-1}\right)\left(t_{1}, t_{2}\right)=0\right. \\
& \wedge \quad y-\operatorname{Im}\left(\wp_{\Lambda}^{-1}\right)\left(t_{1}, t_{2}\right)=0 \\
& \left.\left.\wedge \quad w+\operatorname{Re}\left(G_{\Lambda}\right)\left(t_{1}, t_{2}\right)=0\right)\right\} .
\end{aligned}
$$


By the proof of Lemma 3.1, $\operatorname{Re}\left(\wp_{\Lambda}^{-1}\right), \operatorname{Im}\left(\wp_{\Lambda}^{-1}\right)$ and $\operatorname{Re}\left(G_{\Lambda}\right)$ are Pfaffian functions of common order 8 and common degree $(9,1)$, and it follows that the functions featuring in the above definition are as well, giving the required complexity. An analogous expression can be given for graph $\left(\operatorname{Im}\left(\zeta_{\Lambda}\right)\right)$.

\section{The general case and proof of Theorem 1.3(i)}

For the remainder of this note we fix a lattice $\Lambda$, with fixed generators $\omega_{1}$ and $\omega_{2}$ as above, and set $\mathcal{F}$ to be the fundamental domain with corners $0, \omega_{1}, \omega_{2}$ and $\omega_{1}+\omega_{2}$.

In this setting we fix $k \in \mathbb{N}$ and make use of two results of Masser to obtain a upper bound on $N_{k}\left(\mathcal{W}_{1}, T\right)$, where $\mathcal{W}_{1}$ is again the graph of $\zeta_{\Lambda}$ restricted to a domain which depends on the lattice $\Lambda$, in this case the disc around the point $\frac{\omega_{1}+\omega_{2}}{4}$ of a radius $R$ which will be indicated shortly.

The first result we employ is the following, an immediate consequence of [Mas11, Proposition 2].

Lemma 4.1. For any $k \in \mathbb{N}$ and any real $R>0, S>0, T \geq$ e, let $f_{1}, f_{2}: U \rightarrow$ $\mathbb{C}$ be complex analytic functions on an open neighbourhood $U$ of $\{z \in \mathbb{C}|| z \mid \leq$ $R\}$ such that $\max _{|z| \leq R}\left\{\left|f_{i}(z)\right|\right\} \leq S$, for $i=1,2$. If $\mathcal{Z}$ is a finite set of complex numbers such that, for all $z, z^{\prime} \in \mathcal{Z}$,

(i) $\left(f_{1}(z), f_{2}(z)\right) \in \mathcal{X}(k, T)$, where $\mathcal{X}$ is the image of $f=\left(f_{1}, f_{2}\right)$;

(ii) $|z| \leq \frac{R}{2}$

(iii) $\left|z-z^{\prime}\right| \leq \frac{R}{4}$

then there exists $c(k, S)>0$ such that $f(\mathcal{Z})$ is contained in the zero set of some non-zero $P \in \mathbb{Z}\left[Z_{1}, Z_{2}\right]$ with degree at most $c \cdot \log T$.

Proof. We will apply [Mas11, Proposition 2]. In order to do so, we set the variable $d$ there to be our $k, A$ to be $\frac{4}{R}, Z$ to be $\frac{R}{2}, M$ to be $S, H$ to be our $T$, and the $T$ there to be $c \cdot \log T$ for our $T$. Then the statement above follows from [Mas11, Proposition 2] if

(a) $c \cdot \log T \geq \sqrt{8 k}$ and

(b) $c \geq \frac{142 k^{2}+16 k \log (S+1)}{(\log 2)(\log T)}+\frac{48 k^{2}}{\log 2}$,

for all $T \geq e$. It is clear that one can choose $c>0$ large enough that both of these inequalities can be satisfied together. The fact that the algebraic curve obtained is defined over $\mathbb{Z}$ follows from the proof of [Mas11, Proposition 2].

Choose a real number $R>0$ such that there is an open neighbourhood $U$ of $\Delta_{2 R}:=\left\{z|| z-\frac{\omega_{1}+\omega_{2}}{4} \mid \leq 2 R\right\}$ with $U \subseteq \mathcal{F} \backslash(\Lambda / 2)$. Note that $R$ can be chosen effectively in terms of $\omega_{1}$ and $\omega_{2}$. We set $\Delta_{R}:=\left\{z|| z-\frac{\omega_{1}+\omega_{2}}{4} \mid \leq R\right\}$ and let $\mathcal{W}_{1}$ be the graph of $\left.\zeta_{\Lambda}\right\rceil_{R}$.

Let $N \in \mathbb{N}$ be such that we can cover $\Delta_{R}$ with $N$ closed $\operatorname{discs} \Delta^{(1)}, \ldots, \Delta^{(N)}$ of radius $\frac{R}{8}$. Let $z_{1}, \ldots, z_{N}$ be the corresponding centres of these discs. For each $i \in\{1, \ldots, N\}$, consider the functions $f_{i, 1}, f_{i, 2}: U_{i} \rightarrow \mathbb{C}$ given by

$$
f_{i, 1}(z)=z+z_{i}, \quad f_{i, 2}(z)=\zeta_{\Lambda}\left(z+z_{i}\right)
$$


where $U_{i}$ is the translate $U-z_{i}$. Fix $T \geq 1$. Since $f_{i, 1}, f_{i, 2}$ are analytic on $U_{i}$, we would like to apply Lemma 4.1 to

$$
\mathcal{Z}_{i}:=\left\{z \in D_{\frac{R}{8}} \mid\left(f_{i, 1}, f_{i, 2}\right)(z) \in \operatorname{graph}\left(\zeta_{\Lambda}\left(\Delta_{i}\right)\right)(k, T)\right\},
$$

where $D_{\frac{R}{8}}$ denotes the closed disc of radius $\frac{R}{8}$ around the origin. However, before we do so, we explain how to find real $S_{i}>0$ such that $\max _{|z| \leq R}\left\{\left|f_{i}(z)\right|\right\} \leq S_{i}$, for $i=1,2$.

In order to find such $S_{i}$, it is enough to find a real $S>0$ such that $\max _{z \in \Delta_{2 R}}\left\{|z|,\left|\zeta_{\Lambda}(z)\right|\right\} \leq S$. It is easy to see that $\frac{\left|\omega_{1}+\omega_{2}\right|}{4}+2 R$ is a bound on $\max _{z \in \Delta_{2 R}}\{|z|\}$. To obtain a bound on $\max _{z \in \Delta_{2 R}}\left\{\left|\zeta_{\Lambda}(z)\right|\right\}$, we appeal to the following theorem of Masser.

Fact 4.2 ([Mas03]). For any lattice $\Lambda$ and any $z \in \mathbb{C} \backslash \Lambda$, we have

$$
\left|\wp_{\Lambda}^{\prime}(z)\right| \leq \frac{M}{d(z ; \Lambda)^{3}}
$$

where $d(z ; \Lambda)$ is the distance of $z$ from the lattice $\Lambda$, and $M=\frac{\Gamma\left(\frac{1}{3}\right)^{9}}{(2 \pi)^{3} \cdot 3^{\frac{3}{2}}} \approx 5.5$.

We fix the notation $\delta:=\min _{z \in \Delta_{2 R}} d(z ; \Lambda)$, so $\delta$ depends only on $\omega_{1}, \omega_{2}$. By Fact 4.2 we then have that $\left|\wp_{\Lambda}^{\prime}(z)\right| \leq \frac{M}{\delta^{3}}$.

Now, let $z$ be a point on the boundary of $\Delta_{2 R}$, and let $C$ be the straight line from $\frac{\omega_{1}+\omega_{2}}{4}$ to $z$. Then

$$
\wp_{\Lambda}(z)-\wp_{\Lambda}\left(\frac{\omega_{1}+\omega_{2}}{4}\right)=\int_{C} \wp_{\Lambda}^{\prime}(\xi) d \xi
$$

and so

$$
\begin{aligned}
\left|\wp_{\Lambda}(z)\right| & \leq \int_{C}\left|\wp_{\Lambda}^{\prime}(\xi)\right| d \xi+\left|\wp_{\Lambda}\left(\frac{\omega_{1}+\omega_{2}}{4}\right)\right| \\
& \leq \frac{2 M R}{\delta^{3}}+\left|\wp_{\Lambda}\left(\frac{\omega_{1}+\omega_{2}}{4}\right)\right| .
\end{aligned}
$$

We also have that, for $\operatorname{such} z$,

$$
\zeta_{\Lambda}(z)-\zeta_{\Lambda}\left(\frac{\omega_{1}+\omega_{2}}{4}\right)=\int_{C} \zeta_{\Lambda}^{\prime}(\xi) d \xi
$$

and consequently, since $\zeta_{\Lambda}^{\prime}=-\wp_{\Lambda}$,

$$
\begin{aligned}
\left|\zeta_{\Lambda}(z)\right| & \leq \int_{C}\left|\wp_{\Lambda}(\xi)\right| d \xi+\left|\zeta_{\Lambda}\left(\frac{\omega_{1}+\omega_{2}}{4}\right)\right| \\
& \leq \frac{4 M R^{2}}{\delta^{3}}+2 R\left|\wp_{\Lambda}\left(\frac{\omega_{1}+\omega_{2}}{4}\right)\right|+\left|\zeta_{\Lambda}\left(\frac{\omega_{1}+\omega_{2}}{4}\right)\right| .
\end{aligned}
$$

Therefore, if we set

$$
S:=\max \left\{\frac{\left|\omega_{1}+\omega_{2}\right|}{4}+2 R, \frac{4 M R^{2}}{\delta^{3}}+2 R\left|\wp_{\Lambda}\left(\frac{\omega_{1}+\omega_{2}}{4}\right)\right|+\left|\zeta_{\Lambda}\left(\frac{\omega_{1}+\omega_{2}}{4}\right)\right|\right\},
$$


we have that $\max _{z \in \Delta_{2 R}}\left\{|z|,\left|\zeta_{\Lambda}(z)\right|\right\} \leq S$.

Applying Lemma 4.1 then to each $\mathcal{Z}_{i}$, for $i \in\{1, \ldots, N\}$, and noting that $\# \mathcal{Z}_{i}=\# \operatorname{graph}\left(\zeta_{\Lambda}\left(\Delta^{(i)}\right)\right)(k, T)$, the above calculations tell us that there is an effective constant $c_{0}\left(k, \omega_{1}, \omega_{2}\right)$ such that $\mathcal{W}_{1}(k, T)$ is contained in $N$ algebraic curves over $\mathbb{Z}$ of degree at most $c_{0} \cdot \log T$.

Now we consider $\mathcal{W}_{1} \cap V(Q)$ for an arbitrary $Q \in \mathbb{Z}[X, Y, W, Z]$ of degree $d \leq c_{0} \cdot \log T$. We first identify $\mathbb{C}$ with $\mathbb{R}^{2}$, then follow the argument of [JMT11]. Let $\widetilde{Q} \in \mathbb{Z}\left[X, Y, W, Z, T_{1}, T_{2}\right]$ be given by $\widetilde{Q}\left(X, Y, W, Z, T_{1}, T_{2}\right):=Q(X, Y, W, Z)$. Then $\mathcal{W}_{1} \cap V(Q)$ is given by $\Pi\left(\widetilde{\mathcal{W}}_{1} \cap V(\widetilde{Q})\right)$, where $\Pi: \mathbb{R}^{6} \rightarrow \mathbb{R}^{4}$ is the natural projection map onto the first four coordinates and $\widetilde{\mathcal{W}}_{1}$ is defined by

$$
\begin{aligned}
\widetilde{\mathcal{W}}_{1}:=\left\{\left(x, y, w, z, t_{1}, t_{2}\right) \in \Delta_{R} \times \mathbb{R}^{4} \mid\right. & \\
& x-\operatorname{Re}\left(\wp_{\Lambda}^{-1}\right)\left(t_{1}, t_{2}\right)=0 \\
& \wedge y-\operatorname{Im}\left(\wp_{\Lambda}^{-1}\right)\left(t_{1}, t_{2}\right)=0 \\
& \wedge w+\operatorname{Re}\left(G_{\Lambda}\right)\left(t_{1}, t_{2}\right)=0 \\
& \left.\wedge z+\operatorname{Im}\left(G_{\Lambda}\right)\left(t_{1}, t_{2}\right)=0\right\} .
\end{aligned}
$$

Since $\zeta_{\Lambda} \uparrow_{\Delta_{R}}$ is transcendental, the number of points in the set $\mathcal{W}_{1} \cap V(Q)$ is bounded above by the number of connected components of $\widetilde{\mathcal{W}}_{1} \cap V(\widetilde{Q})$. The latter can be computed first using Lemma 3.1, which tells us that the Pfaffian functions defining $\widetilde{W}_{1} \cap V(\widetilde{Q})$ are of common order 9 and common degree $(9, d)$, where $d$ is at most $c_{0} \cdot \log T$. Then, by Fact 2.3, there is an effective constant $c_{1}\left(k, \omega_{1}, \omega_{2}\right)$ such that the size of the intersection $\mathcal{W}_{1} \cap V(Q)$ is bounded by $c_{1} \cdot(\log T)^{15}$. Consequently, we obtain the following bound:

$$
\begin{aligned}
N_{k}\left(\mathcal{W}_{1}, T\right) & \leq N \cdot c_{1} \cdot(\log T)^{15} \\
& \leq c_{2}\left(k, \omega_{1}, \omega_{2}\right) \cdot(\log T)^{15},
\end{aligned}
$$

for some effective constant $c_{2}>0$.

\section{$5 \quad$ Weierstrass zeta functions on $\mathbb{R}$}

For this section, we fix a real number field $F \subseteq \mathbb{C}$ of degree $k \in \mathbb{N}$ and consider here the two cases of Theorem 1.3 which provide an upper bound on $N_{F}(\mathcal{W}, T)$, where $\mathcal{W}$ is, in each case, the graph of $\zeta_{\Lambda}$ restricted to an appropriate interval within $\mathcal{F} \backslash(\Lambda / 2) \cap \mathbb{R}$.

\subsection{Mild parameterization}

In this case we follow the approach of [JMT11] to find a bound on $N_{F}\left(\mathcal{W}_{2}, T\right)$, where $\mathcal{W}_{2}$ is the graph of $\zeta_{\Lambda} \uparrow_{B}$, for some compact subinterval $B$ of $(0,1) \cap \mathcal{F} \backslash$ $(\Lambda / 2)$. We include the details here as we wish to compute the exponent involved, to enable comparison with the other methods, and to show that the constant is effective. We make use of the following two definitions and subsequent fact, a special case of [Pil10], Corollary 3.3. In order to state these, we require the following multi-index notation: for any $\alpha=\left(\alpha_{1}, \ldots, \alpha_{n}\right) \in \mathbb{N}^{n}$, we define the modulus $|\alpha|:=\alpha_{1}+\ldots+\alpha_{n}$, the factorial $\alpha !:=\alpha_{1} ! \cdot \ldots \cdot \alpha_{n}$ ! and the differential operator

$$
D^{\alpha}:=\frac{\partial^{|\alpha|}}{\partial x_{1}^{\alpha_{1}} \ldots \partial x_{n}^{\alpha_{n}}} .
$$


Definition 5.1. Let $A>0, C \geq 0$. A $C^{\infty}$ function $\phi:(0,1)^{n} \rightarrow(0,1)$ is said to be $(A, C)$-mild if

$$
\left|D^{\alpha} \phi(\bar{x})\right| \leq \alpha !\left(A|\alpha|^{C}\right)^{|\alpha|}
$$

for all $\alpha \in \mathbb{N}^{n}$ and all $\bar{x} \in(0,1)^{n}$. We say that a map $\phi:(0,1)^{n} \rightarrow(0,1)^{m}$ is $(A, C)$-mild if each of its coordinate functions is $(A, C)$-mild.

Definition 5.2. Let $\mathcal{W}$ be a subset of $\mathbb{R}^{n}$. A parameterization of $\mathcal{W}$ is a finite set $\mathcal{S}$ of maps $\phi_{1}, \ldots, \phi_{l}:(0,1)^{\operatorname{dim} \mathcal{W}} \rightarrow \mathbb{R}^{n}$ such that $\mathcal{W}=\bigcup \phi_{i}\left((0,1)^{\operatorname{dim} \mathcal{W}}\right)$. A parameterization is said to be $(A, C)$-mild if each of the parameterizing maps is $(A, C)$-mild.

Fact 5.3. Let $\mathcal{W}$ be a subset of $(0,1)^{n}$ of dimension $\delta$ and suppose that there is an $(A, 0)$-mild parameterization $\mathcal{S}$ of $\mathcal{W}$. There is a constant $c_{3}(n, \delta)>0$ such that $\mathcal{W}(F, T)$ is contained in a union of at most

$$
\# \mathcal{S} \cdot c_{3}^{k} \cdot A^{(\delta+1)(1+o(1))}
$$

intersections of $\mathcal{W}$ with algebraic curves of degree at most $(k \cdot \log T)^{\frac{\delta}{n-\delta}}$. Here the $1+o(1)$ is taken as $T \rightarrow \infty$ with absolute implied constant.

Let $B$ be a compact interval in $(0,1) \cap \mathcal{F} \backslash(\Lambda / 2)$ and let $\mathcal{W}_{2} \subseteq \mathbb{C}^{2}$ be the graph of $\zeta_{\Lambda} \uparrow_{B}$. We let $K$ be a Galois closure of $F$ and note that if $x+i y, w+i z \in F$, with $x, y, w, z \in \mathbb{R}$, then $x, y, w, z \in K$. In particular, for each point $(x, w+i z)$ on $\mathcal{W}_{2}(F, T)$, there is a unique point $(x, w)$ on $\mathcal{W}_{2}^{\prime}(K, T)$, the graph of the real function $\operatorname{Re}\left(\zeta_{\Lambda} \uparrow_{B}\right): B \rightarrow \mathbb{R}$. Therefore, in order to bound $N_{F}\left(\mathcal{W}_{2}, T\right)$, it is enough to bound $N_{K}\left(\mathcal{W}_{2}^{\prime}, T\right)$.

Now we note that the inversions $z \mapsto \pm z^{ \pm 1}$ preserve both the complexity of definition from Pfaffian functions and the collection of algebraic points of height bounded by $T$ and degree bounded by $k$. Therefore, let us reduce to considering the case that $\operatorname{Re}\left(\zeta_{\Lambda}(B)\right)$ is also contained in $[0,1]$. The result without this assumption may then be derived from this special case by multiplying through by a factor of $c_{4}(B)>0$, a constant depending on the number of times $\zeta_{\Lambda} \Upsilon_{B}$ takes the values $-1,0,1$, which can be computed only using $B$ and the Pfaffian complexity of $\operatorname{Re}\left(\zeta_{\Lambda} \uparrow_{B}\right)$.

Since $\zeta_{\Lambda}$ is analytic on an open set containing $B$, it is mild on $B$. Moreover, the function $\operatorname{Re}\left(\zeta_{\Lambda} \Gamma_{B}\right)$ is mild, and this is the property that we shall use. Since it is a mild function, it is an $(A, 0)$-mild parameterization of itself, where $A$ depends on the lattice (i.e. on the lattice generators $\omega_{1}$ and $\omega_{2}$ ) and can be found using the methods of Section 4. By Fact 5.3 above, there exists an absolute constant $c_{3}$ such that $\mathcal{W}_{2}^{\prime}(K, T)$ is contained in $c_{3}^{k} \cdot A^{2(1+o(1))}$ intersections of $\mathcal{W}_{2}^{\prime}$ with sets of the form $\left\{(x, w) \in \mathbb{R}^{2} \mid P(x, w)=0\right\}$, where $P \in \mathbb{R}[X, W]$ is a polynomial of degree at most $k ! \cdot \log T$ (the degree of $K$ is bounded by $k !$ ).

Now let us consider such an intersection for a given polynomial $P$. We proceed as before, following the argument of [JMT11], and let $\widetilde{P} \in \mathbb{R}\left[X, W, T_{1}, T_{2}\right]$ be given by $\widetilde{P}\left(X, W, T_{1}, T_{2}\right):=P(X, W)$. Then $\mathcal{W}_{2}^{\prime} \cap V(P)$ is given by $\Pi\left(\widetilde{\mathcal{W}}_{2}^{\prime} \cap\right.$ $V(\widetilde{P}))$, where $\Pi$ is the projection map given above, and $\widetilde{\mathcal{W}_{2}^{\prime}}$ is defined by

$$
\begin{aligned}
\widetilde{\mathcal{W}_{2}^{\prime}}:=\left\{\left(x, w, t_{1}, t_{2}\right) \in B \times(0,1) \times \mathbb{R}^{2} \mid\right. & x-\operatorname{Re}\left(\wp_{\Lambda}^{-1}\right)\left(t_{1}, t_{2}\right)=0 \\
& \wedge \operatorname{Im}\left(\wp_{\Lambda}^{-1}\right)\left(t_{1}, t_{2}\right)=0 \\
& \left.\wedge w+\operatorname{Re}\left(G_{\Lambda}\right)\left(t_{1}, t_{2}\right)=0\right\} .
\end{aligned}
$$


As before, since $\left.\zeta_{\Lambda}\right\rceil_{B}$ is transcendental, the number of points in the set $\mathcal{W}_{2}^{\prime} \cap$ $V(P)$ is bounded above by the number of connected components of $\widetilde{\mathcal{W}_{2}^{\prime}} \cap V(\widetilde{P})$. In this case, the proof of Lemma 3.1 tells us that the Pfaffian functions defining $\widetilde{\mathcal{W}_{2}^{\prime}} \cap V(\widetilde{P})$ are of common order 8 and common degree $(9, d)$, where $d:=\operatorname{deg} P$. Then, by applying Fact 2.3, we see that the size of the intersection $\mathcal{W}_{2}^{\prime} \cap V(P)$ is bounded by $c_{5} \cdot d^{12}$, where $c_{5}>0$ is an absolute constant. Consequently, we obtain the following bound (using $d \leq k ! \cdot \log T$ ):

$$
\begin{aligned}
N_{F}\left(\mathcal{W}_{2}, T\right) \leq N_{K}\left(\mathcal{W}_{2}^{\prime}, T\right) & \leq c_{4} \cdot c_{5} \cdot(k ! \cdot \log T)^{12} \cdot c_{3}^{k !} \cdot A^{2(1+o(1))} \\
& \leq c_{6}\left(k, B, \omega_{1}, \omega_{2}\right) \cdot(\log T)^{12}
\end{aligned}
$$

\subsection{Implicitly defined from Pfaffian functions}

In the previous subsection the bound obtained on $N_{F}\left(\mathcal{W}_{2}, T\right)$, where $\mathcal{W}_{2}$ is the graph of $\left.\zeta_{\Lambda}\right|_{B}$, for some compact $B \subseteq(0,1) \cap \mathcal{F} \backslash(\Lambda / 2)$, is of the form $c \cdot(\log T)^{\gamma}$, where $\gamma=12$ and the constant $c$ depends not only on $k$ but also on $A$, i.e. on the lattice $\Lambda$.

We may, however, use a different result to obtain a bound of the same form on $N_{F}\left(\mathcal{W}_{3}, T\right)$, where $\mathcal{W}_{3}$ is the graph of $\left.\zeta_{\Lambda}\right|_{I}$, for $I:=\mathbb{R} \cap \mathcal{F}$, with the constant $c$ depending only on $k$; in other words, $c$ does not depend on the lattice. This is achieved at the cost of increasing the exponent $\gamma$. The result which provides this bound is the following.

Fact 5.4 ([JT12, 4.3]). Let $I$ be an open interval in $\mathbb{R}$ and $\phi: I \rightarrow \mathbb{R}$ a transcendental function which is implicitly definable in $\mathbb{R}_{\text {Pfaff }}$ with complexity $(n, r, \alpha, \beta)$. There are explicit constants $c_{7}(k), c_{8}(n, r, \alpha, \beta)$ such that, for $T \geq e$,

$$
N_{F}(\mathcal{W}, T) \leq c_{7} \cdot c_{8} \cdot(\log T)^{3 n+3 r+8},
$$

where $\mathcal{W}$ is the graph of $\phi$.

Consider the set $I \backslash(\Lambda / 2)$, which is the union of at most two intervals. For each such subinterval $J$, the function $\operatorname{Re}\left(\zeta_{\Lambda} \uparrow_{J}\right)$ is implicitly defined from Pfaffian functions with complexity $(3,8,9,1)$, by Corollary 3.2 . Hence, applying Fact 5.4 on each subinterval, and noting that there is at most one additional point on $\mathcal{W}_{3}$ not appearing on the graph of some $\zeta_{\Lambda} \uparrow_{J}$, we see that $N_{F}\left(\mathcal{W}_{3}, T\right) \leq$ $c_{9}(k) \cdot(\log T)^{41}$.

\section{References}

[Bes14] Etienne Besson. Points rationnels de la fonction Gamma d'Euler. Arch. Math. (Basel), 103(1):61-73, 2014.

[BG06] Enrico Bombieri and Walter Gubler. Heights in Diophantine geometry, volume 4 of New Mathematical Monographs. Cambridge University Press, Cambridge, 2006.

[BJ15] Gareth J. Boxall and Gareth O. Jones. Algebraic values of certain analytic functions. Int. Math. Res. Not. IMRN, (4):1141-1158, 2015. 
[DV73] Patrick Du Val. Elliptic functions and elliptic curves. Cambridge University Press, London, 1973. London Mathematical Society Lecture Note Series, No. 9.

[GV04] Andrei Gabrièlov and Nicolai Vorobjov. Complexity of computations with Pfaffian and Noetherian functions. In Normal forms, bifurcations and finiteness problems in differential equations, volume 137 of NATO Sci. Ser. II Math. Phys. Chem., pages 211-250. Kluwer Acad. Publ., Dordrecht, 2004.

[JMT11] Gareth O. Jones, Daniel J. Miller, and Margaret E. M. Thomas. Mildness and the density of rational points on certain transcendental curves. Notre Dame J. Form. Log., 52(1):67-74, 2011.

[JT12] Gareth O. Jones and Margaret E. M. Thomas. The density of algebraic points on certain Pfaffian surfaces. Q. J. Math., 63(3):637-651, 2012.

[JW08] G. O. Jones and A. J. Wilkie. Locally Polynomially Bounded Structures. Bull. London Math. Soc., 40:239-248, 2008.

[Kho80] A. G. Khovanskii. A class of systems of transcendental equations. Dokl. Akad. Nauk SSSR, 255(4):804-807, 1980.

[Kho91] A. G. Khovanskii. Fewnomials, volume 88 of Translations of Mathematical Monographs. American Mathematical Society, Providence, RI, 1991. Translated from the Russian by Smilka Zdravkovska.

[Mac08] Angus Macintyre. Some observations about the real and imaginary parts of complex Pfaffian functions. In Model theory with applications to algebra and analysis. Vol. 1, volume 349 of London Math. Soc. Lecture Note Ser., pages 215-223. Cambridge Univ. Press, Cambridge, 2008.

[Mas03] D. W. Masser. Sharp estimates for Weierstrass elliptic functions. J. Anal. Math., 90:257-302, 2003.

[Mas11] D. Masser. Rational values of the Riemann zeta function. J. Number Theory, 131(11):2037-2046, 2011.

[Pil10] Jonathan Pila. Counting rational points on a certain exponentialalgebraic surface. Ann. Inst. Fourier (Grenoble), 60(2):489-514, 2010. 